\title{
OXYGEN METALLICITY DETERMINATIONS FROM OPTICAL EMISSION LINES IN EARLY-TYPE GALAXIES
}

\author{
Alex E. Athey ${ }^{1}$ and Joel N. Bregman ${ }^{2}$ \\ ${ }^{1}$ The University of Texas at Austin, Applied Research Laboratories, 10000 Burnet Rd., Austin, TX 78758, USA; athey@ arlut.utexas.edu \\ ${ }^{2}$ University of Michigan, Department of Astronomy, 500 Church Street, Ann Arbor, MI 48109-1090, USA; jbregman@umich.edu \\ Received 2008 May 23; accepted 2009 February 9; published 2009 April 17
}

\begin{abstract}
We measured the oxygen abundances of the warm $\left(T \sim 10^{4} \mathrm{~K}\right)$ phase of gas in seven early-type galaxies through long-slit observations. A template spectra was constructed from galaxies void of warm gas and subtracted from the emission-line galaxies, allowing for a clean measurement of the nebular lines. The ratios of the emission lines are consistent with photoionization, which likely originates from the ultraviolet flux of postasymototic giant branch stars. We employ H II region photoionization models to determine a mean oxygen metallicity of $1.01 \pm 0.50$ solar for the warm interstellar medium (ISM) in this sample. This warm ISM $0.5-1.5$ solar metallicity is consistent with modern determinations of the metallicity in the hot $\left(T \sim 10^{6}-10^{7} \mathrm{~K}\right)$ ISM and the upper range of this warm ISM metallicity is consistent with stellar population metallicity determinations. A solar metallicity of the warm ISM favors an internal origin for the warm ISM such as asymptotic giant branch mass loss within the galaxy.
\end{abstract}

Key words: galaxies: abundances - galaxies: elliptical and lenticular, $\mathrm{cD}$ - galaxies: ISM

\section{INTRODUCTION}

\subsection{Warm ISM Discovery}

Early-type galaxies were once thought to contain very little gas in their interstellar medium (ISM; e.g., Mathews \& Baker 1971; Bregman 1978; White \& Chevalier 1983), but are now known to host massive amounts of extremely hot $\left(T \sim 10^{6}-10^{7} \mathrm{~K}\right.$ ) gas that emits in the X-rays (Forman et al. 1979). The reason early-type galaxies were originally thought to be void of gas is that photographic-plate surveys did not reveal the warm $\left(T \sim 10^{4} \mathrm{~K}\right)$ ISM that is abundant in spiral galaxies (e.g., Mayall 1936; Sandage 1957; Mayall 1958). Only with the advent of large telescopes and efficient detectors was the warm ISM detected, although the masses are an order of magnitude lower than that of spirals. Spectroscopic surveys in the mid-1980s determined warm ISM detection frequencies in the cores of early-type galaxies of 30\%-50\% (Caldwell 1984; Phillips et al. 1986; Veron-Cetty \& Veron 1986). Subsequent narrowband $\mathrm{H} \alpha$ imaging studies revealed extended emission in general agreement with the stellar morphology and detection rates consistent with the $50 \%$ rate determined from the early spectroscopic surveys (Demoulin-Ulrich et al. 1984; Kim 1989).

\subsection{Key Warm ISM Studies}

There are three large sample, multiwavelength studies of early-type galaxies that have provided a foundation for our understanding of the cold and warm ISM. Trinchieri \& di Serego Alighieri (1991, hereafter TdSA) conducted one of the first quantitative early-type galaxy emission-line studies with $\mathrm{H} \alpha$ imaging and long-slit optical spectroscopy of 13 earlytype galaxies known to have hot X-ray halos. Goudfrooij et al. (1994a, 1994b, 1994c) and Goudfrooij \& de Jong (1995, hereafter G94-5) investigated the origin and evolution of the ISM in an extensive study of a complete sample of 56 elliptical galaxies using broadband imaging, narrowband $\mathrm{H} \alpha$ imaging, long-slit spectroscopy, and Infrared Astronomical Satellite (IRAS) farinfrared (FIR) imaging. A study of the warm and cold phases of the ISM in early-type galaxies was carried as part of the ESO Key-Program 1-004-43K (Macchetto et al. 1996; Ferrari et al. 1999; Caon et al. 2000; Ferrari et al. 2002, hereafter MFCF). Similar to G94-5, the MFCF study probed the origin and evolution of the warm and cold ISM phases through a combination of broadband, narrowband, and FIR imaging and long-slit spectroscopy of 73 early-type galaxies. The two later studies had the benefit of FIR imaging capabilities and added the cold ISM (i.e., dust) to the discussion of the ISM in early-type galaxies. These studies sought to determine the frequency of detection of warm and cold ISM in early-type galaxies and then measure fundamental properties, such as mass, density, and luminosity. By correlating these properties with stellar and hot ISM properties, inferences are made about the origin and evolution of the ISM and how it relates to the origin and evolution of the galaxy itself. Below we briefly summarize the findings of these key studies and note the areas where the studies agree and where questions remain.

The three key studies find similar fractions of their samples that host a warm ISM, ranging from $60 \%$ to $80 \%$, despite the differences in sample selection criteria and sizes of the samples. TdSA found $75 \%$ of the 13 early-type galaxies surveyed had detectable $\mathrm{H} \alpha$ emission which was extended over a range of 5$10 \mathrm{kpc}$. The extent and elliptical shape of the emission confirmed the galaxy-wide nature of this phenomena and excluded a nuclear confined process, such as active galactic nucleus (AGN) activity. The TdSA survey was conducted on galaxies known to have significant masses of hot ISM, and the authors concede that the high detection rate is likely connected to sample selection. The G94-5 optically complete sample (chosen without regard for X-ray luminosity) offers a less biased measure of the frequency of the warm ISM; $60 \%$ of the early-type galaxies surveyed were detected to have a warm ISM, while a cold $(T \sim 10 \mathrm{~K})$ dust component is detected in $40 \%$ of these galaxies. Through the FIR imaging, the G94-5 study was able to show that unlike spiral galaxies, early-type galaxies display a smooth dust component distributed throughout the galaxy. The MFCF authors report an ionized gas detection frequency at $78 \%$ for the 73 galaxies in the optically complete sample. The cold dust 
component was detected at a $75 \%$ detection rate with smooth, extended morphologies similar to that of the warm ISM. Two key observations link the warm and the cold ISM together: first, the similar spatial extent and morphology of the cold ISM (dust) and warm ISM, and second the fact that the presence of dust is a strong predictor of the presence of emission lines. It is concluded that these ISM phases are closely related and likely of the same origin.

The mass of the warm ISM is orders of magnitude below the X-ray emitting hot ISM and the stellar mass. TdSA calculated a warm ISM gas mass by assuming case-B recombination, a filling factor of $10^{-5}-10^{-6}$, and densities corresponding to the pressure implied by the X-ray observations. The resultant masses were calculated to be $\sim 10^{5}-10^{6} M_{\odot}$ for a typical galaxy in their sample. The $\mathrm{H} \alpha$ imaging in G94-5 revealed warm ISM masses similar to the FIR-determined dust masses of $\sim 10^{4}-10^{5} M_{\odot}$. The MFCF study determined masses for the warm ISM in the range of $10^{3}-10^{5} M_{\odot}$. Masses of dust at $10^{5} M_{\odot}$ have direct implications on the origin of the dust as the dust destruction rate is high in the presence of the hot ISM and is further discussed below.

The correlations between the luminosities of $L_{\mathrm{FIR}}, L_{\mathrm{H} \alpha}, L_{\mathrm{X}}$, $L_{\mathrm{B}}$, and $L_{\mathrm{X}} / L_{\mathrm{B}}$ explore the links between the difference phases of the ISM and the stars. For numerous reasons, the luminosity correlations have been difficult to accurately measure and there are contradictions between the key studies. TdSA observed that galaxies with more massive X-ray halos had stronger line emission, indicating that the warm gas is somehow linked with the hot gas. No other luminosity correlations were observed. In a related work to G94-5, the authors find a strong anticorrelation between the masses of dust and X-ray gas (Goudfrooij 1994, 1995). However in a later study with a larger sample (based in part on MFCF data), Goudfrooij (1997) reveals a positive correlation between the warm ISM with both the hot ISM and the stellar blue luminosity. MFCF determined the luminosity of the ionized gas to be correlated with both blue and X-ray luminosity. The scatter in the $L_{\mathrm{H} \alpha}$ and $L_{\mathrm{B}}$ relation is greatly reduced when the blue luminosity is confined to the same region as the emission lines. The scatter in all the $L_{\mathrm{H} \alpha}$ to $L_{\mathrm{X}} / L_{\mathrm{B}}$ relations is large and plagued by upper limits on nondetections.

In general, the three studies agree that the ionization source for the warm ISM is consistent with postasymototic giant branch (PAGB) stars, but all comment that the data are unable to exclude other excitation sources such as internal shocks, AGN heating, or electron conduction from the hot gas. TdSA show that the observed line intensities are a factor of $\sim 10^{2}-10^{3}$ too large to result from cooling flow luminosities (e.g., Fabian 1991) and conclude that ultraviolet (UV) flux from PAGB stars are the most likely ionizing mechanism. The MFCF observed correlation between $\mathrm{H} \alpha$ and blue luminosity is an indication of photoionization of the warm ISM from PAGB stars. However, the observed correlation with X-ray luminosity is consistent with warm ISM excitation via electron conduction from the hot ISM (Sparks et al. 1989); Neither ionization mechanism is preferred in MFCF's large sample of 73 galaxies (see Section 4.1.1 for a continued discussion on the warm ISM ionization source).

The key studies narrow down the origin of this dust and warm gas to either be mass shed from asymptotic giant branch (AGB) stars or the result of small galaxy accretion and mergers. G94-5 reasons that cold dust originated in the circumstellar envelopes of AGB stars are quickly sputtered away by the hot ISM with a typical dust grain lifetime $\left(\sim 10^{7}\right.$ years $)$ much shorter than the age of the galaxy (Draine \& Salpeter 1979). And further, the balance between the dust injection and destruction rate does not lead to the dust masses inferred by the FIR imaging (Faber \& Gallagher 1976; Knapp et al. 1992). The surprising result from the confluence of the G94-5 studies is that the majority of the dust in elliptical galaxies is external in origin. Since the distribution of the ionized gas (i.e., the warm ISM) is observed to be similar to the dust morphology, it is presumed that the warm ISM is likewise external to the galaxy, and will only be seen in galaxies that either have recent merger activity or significant inflows. The high detection frequency of the warm ISM implies that mergers and smaller galaxy acquisitions are common in early-type galaxies. Adding to the puzzle, in a subsample of the gas-rich galaxies, the warm ISM was frequently observed to be kinematically distinct from the main stellar population, also strongly suggesting an external origin for this gas (Caon et al. 2000).

\subsection{Warm ISM Open Issues}

The warm ISM is important to study in early-type galaxies and despite the ambitious efforts of the studies described above and subsequent works, several fundamental questions remain. It is critical to resolve these open issues since the intertwined relationships between the cold, warm, and hot gas phases and the stellar population ultimately define galaxy evolution. Even though the warm ISM mass is orders of magnitude less than the stellar mass, discriminating between internal versus external origin of this material provides pivotal data in formation models. Also, even though minute in mass compared to the cold and hot phases of the ISM, the optical line emissions of the warm ISM can locally dominate the energy output for a region. Finally, the optical emission lines of the warm ISM provides information on the heating and cooling in these systems which demands further exploration since the observed hot ISM properties along with the nondetection of cooling flows creates a "cooling crisis."

The metallicity of the warm ISM is unknown. Because the metallicities of the hot ISM and the stellar metallicities (see Trager et al. 2000b, 2000a) are known, determining the metallicity of the warm ISM can create a link between the gas phases and the stellar population. Also, the metallicity of the warm ISM contains important discriminating information concerning the outstanding issues that the key warm ISM studies were not able to resolve. Specifically, the line ratios of the ionization species provide information on the excitation mechanism of the gas; currently, both electron conduction and photoionization remain viable excitation mechanism.

Also the metallicity of the warm ISM contains clues to the internal versus external origin debate. The gas injected into the ISM from stellar mass loss will generally be more metal rich than gas accreted from dwarf galaxies (see Lequeux et al. 1979; Aaronson 1986).

Because of the important information contained in the metallicity of the warm ISM and the unresolved issues concerning its nature and origin, we have conducted a program to determine the oxygen metallicity of the $T \sim 10^{4} \mathrm{~K}$ gas in a small sample of early-type galaxies. Section 2 describes the sample and observations. The data processing techniques and spectral analysis are discussed in Section 3. Results from the emission-line analysis is presented in Section 4. In Section 5, we conclude with a discussion of the implications of this work on both the origin of the warm ISM and the relationships between the stellar population and the other phases of the ISM. 
Table 1

Early-type Galaxy Properties: Warm Phase Sample

\begin{tabular}{|c|c|c|c|c|c|c|c|c|}
\hline Galaxy & $\begin{array}{c}\text { R.A. }^{\mathrm{a}} \\
(\mathrm{J} 2000.0)\end{array}$ & $\begin{array}{c}\text { Dec. }^{\mathrm{a}} \\
(\mathrm{J} 2000.0)\end{array}$ & $B^{0} \mathrm{~T}^{\mathrm{b}}$ & $\begin{array}{c}D^{\mathrm{b}} \\
\left(\mathrm{km} \mathrm{s}^{-1}\right)\end{array}$ & $\log L_{\mathrm{B}} / L_{\odot}^{\mathrm{b}}$ & $\begin{array}{c}\log L_{X}{ }^{c} \\
\log \left(\operatorname{erg~s}^{-1}\right)\end{array}$ & $\log L_{\mathrm{H} \alpha}{ }^{\mathrm{d}}$ & $\mathrm{H} \alpha$ Reference \\
\hline NGC 1407 & 034011.8 & -183448 & 10.57 & $1990 \pm 187$ & 11.16 & 41.34 & 39.32 & M96 \\
\hline NGC 1600 & 043139.8 & -050510 & 11.79 & $4019 \pm 489$ & 10.67 & $40.84^{\mathrm{f}}$ & 40.15 & M96 \\
\hline NGC 2768 & 091137.5 & +600215 & 10.93 & $1532 \pm 325$ & 10.79 & 40.41 & $\ldots$ & $\ldots$ \\
\hline NGC 3115 & 100513.9 & -074307 & 9.95 & $1021 \pm 215$ & 10.83 & 39.74 & $<37.6$ & M96 \\
\hline NGC 3377 & 104742.3 & +135908 & 11.13 & $857 \pm 126$ & 10.21 & 39.42 & 38.95 & G94 \\
\hline NGC 3379 & 104749.6 & +123455 & 10.43 & $857 \pm 126$ & 10.49 & 39.78 & 39.04 & M96 \\
\hline NGC 3489 & 110018.3 & +135405 & $11.12^{\mathrm{g}}$ & $1039 \pm 101^{\mathrm{g}}$ & $10.35^{\mathrm{g}}$ & $\cdots$ & 39.34 & M96 \\
\hline NGC 3607 & 111654.3 & +180310 & 10.53 & $1991 \pm 242$ & 11.18 & 40.82 & 39.92 & M96 \\
\hline NGC 4125 & 120805.8 & +651027 & 10.58 & $1986 \pm 295$ & 11.16 & 41.01 & 40.30 & T91 \\
\hline NGC 4261 & 124202.4 & +113848 & 11.32 & $2783 \pm 590$ & 10.35 & $41.18^{\mathrm{g}}$ & 39.38 & G94 \\
\hline NGC 4374 & 122503.7 & +125313 & 10.13 & $1333 \pm 71$ & 10.99 & 41.09 & 39.56 & G94 \\
\hline NGC 4406 & 122611.7 & +125646 & 9.87 & $1333 \pm 71$ & 11.10 & 41.80 & 40.50 & T91 \\
\hline NGC 4472 & 122946.8 & +080002 & 9.32 & $1333 \pm 71$ & 11.32 & 41.77 & 39.60 & T91 \\
\hline NGC 4494 & 123124.1 & +254628 & 10.69 & $695 \pm 147$ & 10.20 & 39.28 & $\ldots$ & $\cdots$ \\
\hline NGC 4552 & 123539.8 & +123323 & 10.84 & $1333 \pm 71$ & 10.71 & 40.92 & 39.26 & M96 \\
\hline NGC 4636 & 124250.0 & +024117 & 10.20 & $1333 \pm 71$ & 10.96 & 41.81 & 39.69 & M96 \\
\hline NGC 4649 & 124339.6 & +113309 & 9.77 & $1333 \pm 71$ & 10.96 & 41.48 & 39.83 & T91 \\
\hline NGC 4697 & 124835.9 & -054802 & 10.03 & $794 \pm 168$ & 10.58 & 40.13 & 39.63 & G94 \\
\hline NGC 5044 & 131523.9 & -162308 & 11.25 & $2982 \pm 314$ & 10.34 & 42.39 & 40.73 & M96 \\
\hline NGC 5322 & 134915.2 & +601126 & 11.09 & $1661 \pm 352$ & 10.80 & 40.11 & 39.74 & G94 \\
\hline NGC 5846 & 150629.2 & +013621 & 10.67 & $2336 \pm 284$ & 11.26 & 42.01 & 40.25 & M96 \\
\hline
\end{tabular}

Notes.

${ }^{a}$ Values taken from NED (NASA/IPAC Extragalactic Database).

b Radial velocity distance corrected for local flows from Faber et al. (1989) unless otherwise noted.

c X-ray luminosity from Brown \& Bregman (1998) unless otherwise noted.

${ }^{\mathrm{d}} \mathrm{H} \alpha$ narrowband imaging luminosity.

${ }^{\mathrm{e}}$ Reference for $\mathrm{H} \alpha$ narrowband imaging luminosity with the following abbreviations: M96, Macchetto et al. (1996); G, Goudfrooij et al. (1994b); and T91, Trinchieri \& di Serego Alighieri (1991).

${ }^{f}$ O'Sullivan et al. (2001).

g RC3, de Vaucouleurs et al. (1991).

\section{OBSERVATIONS}

The Michigan-Dartmouth-MTT $2.4 \mathrm{~m}$ Hiltner Telescope coupled with a Boller and Chivens spectrograph was used to observe 21 nearby, early-type galaxies in four observation runs over a two-year period from 2000 to 2002 . The sample was selected to span a range of hot ISM properties as determined from the Brown \& Bregman (1998) X-ray study as well as a range of warm ISM properties determined in the narrowband $\mathrm{H} \alpha$ imaging surveys by TdSA, G94-5, and MFCF. The galaxies with their relevant fundamental properties are listed in Table 1. Our warm ISM isolation and detection method depends on observing galaxies that contain no emission-line gas, therefore, several galaxies were chosen to have very weak detections of the hot and warm phases.

The configuration of the spectrograph was chosen to maximize galaxy light input with a $2^{\prime \prime} .1$ wide $\times 5^{\prime}$ long slit, while retaining the ability to discriminate between the $\mathrm{H} \alpha$ and $\left[\mathrm{N}_{\mathrm{II}}\right] \lambda 6583$ emission lines. The available and appropriate grating for these requirements was a 350 lines $\mathrm{mm}^{-1}$ grating blazed at $4026 \AA$, resulting in $7.1 \AA$ pixel $^{-1}$ and a spectral range of $\sim 1600$ A over the $1200 \times 800$ pixel Loral CCD. The CCD was characterized by relatively low read noise at seven electrons with the nominal gain set at 2.1 electrons per ADU. The one drawback to the chosen spectrograph configuration is that it was necessary to obtain separate blue and red spectra for each galaxy in order to obtain all of the important emission lines from [O II] $\lambda 3727$ to $[\mathrm{Si}$ II] $\lambda \lambda 6717,6731$. The grating is servo controlled from the control computer and our tests indicated a
$5 \AA$ accuracy in repositioning. Therefore, internal $\mathrm{HgNe}$ and $\mathrm{Ne}$ lamps were observed before and after each grating reposition for wavelength calibration. Additional calibrations included bias frames, evening and morning twilight flats when the skies were clear, internal flats illuminated from an incandescent source, and spectrophotometric standards.

The four observation runs were in 2000 March, 2000 May, 2001 February, and 2002 February. Galaxy exposure times and program-type (E: emission line, T: Template, or L: LINER) are listed in Table 2. The meaning of the program types is discussed below. During the first three runs, the nights were cloudy and over half of the full run was lost to weather and instrument problems. Some of these observations are of marginal value and only reported here for completeness. The final run was entirely photometric.

The observing strategy was chosen to suppress spurious emission line detections and obtain uniform observations of program and template galaxies. For each galaxy, we attempted to obtain five integrations, ensuring that reasonable statistics could be employed to eliminate cosmic rays from the combined data. Because the emission lines are faint and spread over only a few pixels in both spectral and spatial directions, a fortuitous single cosmic ray can result in a spurious warm ISM detection. The total integration time was chosen to result in an $\mathrm{S} / \mathrm{N}$ of $5-10$ in the template subtracted spectra (see Section 3). We did not constrain the angle of the slit due to complications with the instrument rotator during the first two runs; this has little programatic impact because of the choice of a wide slit, and the nonpreferential orientation of the warm ISM 


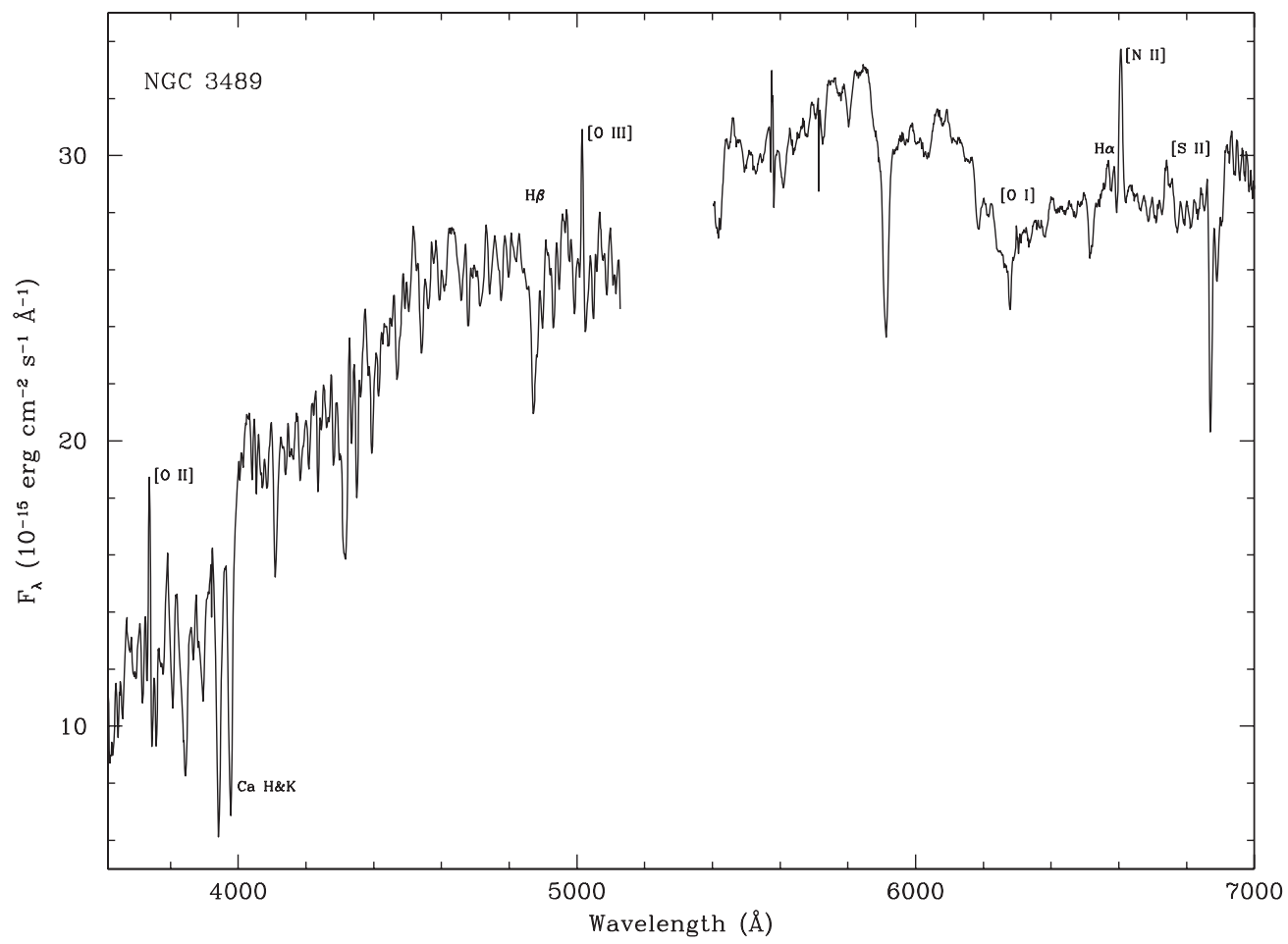

Figure 1. Composite blue and red spectra of NGC3489. Important emission lines that are visible in the unsubtracted data are marked above the continuum level. In addition, calcium $\mathrm{H} \& \mathrm{~K}$ lines are marked for reference.

Table 2

MDM 2.4 m Observations

\begin{tabular}{lccc}
\hline \hline Galaxy & Exp Blue (s) & Exp Red (s) & Emission Type $^{\mathrm{a}}$ \\
\hline NGC 1407 & 8400 & 6600 & $\mathrm{~T}$ \\
NGC 1600 & 8400 & 5400 & $\mathrm{~W}$ \\
NGC 2768 & 12000 & 11400 & $\mathrm{~L}$ \\
NGC 3115 & 6000 & 4500 & $\mathrm{~T}$ \\
NGC 3377 & 7200 & 6000 & $\mathrm{~W}$ \\
NGC 3379 & 6000 & 6000 & $\mathrm{~T}$ \\
NGC 3489 & 6000 & 4500 & $\mathrm{E}$ \\
NGC 3607 & 9600 & 7200 & $\mathrm{E}$ \\
NGC 4125 & 12000 & 10800 & $\mathrm{~L}$ \\
NGC 4261 & 6000 & 4500 & $\mathrm{E}$ \\
NGC 4374 & 6000 & 4500 & $\mathrm{E}$ \\
NGC 4406 & 9600 & 6000 & $\mathrm{~W}$ \\
NGC 4472 & 9600 & 6000 & $\mathrm{~W}$ \\
NGC 4494 & 1200 & 0 & $\mathrm{~W}$ \\
NGC 4552 & 6000 & 6600 & $\mathrm{~W}$ \\
NGC 4636 & 6000 & 9900 & $\mathrm{E}$ \\
NGC 4649 & 6000 & 4500 & $\mathrm{~W}$ \\
NGC 4697 & 6000 & 1800 & $\mathrm{~W}$ \\
NGC 5044 & 6000 & 3600 & $\mathrm{E}$ \\
NGC 5322 & 9600 & 9900 & $\mathrm{~W}$ \\
NGC 5846 & 7200 & 6600 & $\mathrm{E}$
\end{tabular}

Note. ${ }^{\mathrm{a}}$ Emission Type. The column displays how the observations were ultimately used: $\mathrm{T}$, template galaxy; L, LINER galaxy; E, emission galaxy; and $\mathrm{W}$, weak emission.

observed in the narrowband imaging surveys (TdSA; G94-5; MFCF).

\section{REDUCTIONS AND SPECTRAL ANALYSIS}

The data were reduced in the standard manner using tasks within IRAF (overscan fitting and subtraction, bias frame construction and subtraction). Each of the runs was calibrated separately but in a similar manner. Internal lamps were used to create a response image and divided through the data, eliminating differences in pixel-to-pixel sensitivity on the CCD. Twilight flats were used to construct an illumination image, correcting for large scale structure and slit illumination. For the first two runs, the incandescent internal lamp produced too few photons in the far blue and only added noise to the data. For these runs we used wavelength calibrated twilight flats to produce response images. A HgNe lamp was used for wavelength calibration of blue-tuned observations and a $\mathrm{Ne}$ lamp was used for red-tuned observations. Residuals in the linear, spectral solution were much better (typically $<1 \AA$ ) than the resolution element $(\sim 8 \AA)$ over the entire chip.

The galaxies were summed over the spatial dimension based on the visual inspection of the two-dimensional spectra, with background regions selected from the outer regions of the chip. Typically the summed regions for the galaxies were about 1.2 of the 5.2 unvignetted slit length. The spectra were flux calibrated from observations of spectrophotometric standards (Massey et al. 1988) and corrected for extinction with the standard correction for Kitt Peak distributed with IRAF 2.11.

Custom code was developed to implement the combining of the individual one-dinemsional spectra, template matching and subtraction, as well as the extraction of line fluxes. The one-dinemsional spectra combination procedure employed an iterative $1.5 \sigma$ rejection algorithm which eliminated cosmic rays that were overlooked in the aperture summing rejection methods. The wavelength calibration was tuned to night sky lines and the measured redshift was checked and tuned to strong stellar population lines (e.g., $\mathrm{Ca} \mathrm{H} \& \mathrm{~K}$ in the blue and $\left[\mathrm{N}_{\mathrm{II}}\right] \lambda 6583$ in galaxies showing emission lines in the red). An example of summed spectra is shown in Figure 1.

Although we have upper limits from the $\mathrm{H} \alpha$ imaging surveys, it is unknown which galaxies are void of warm ISM emission. Therefore, the determination of template galaxies, or galaxies 

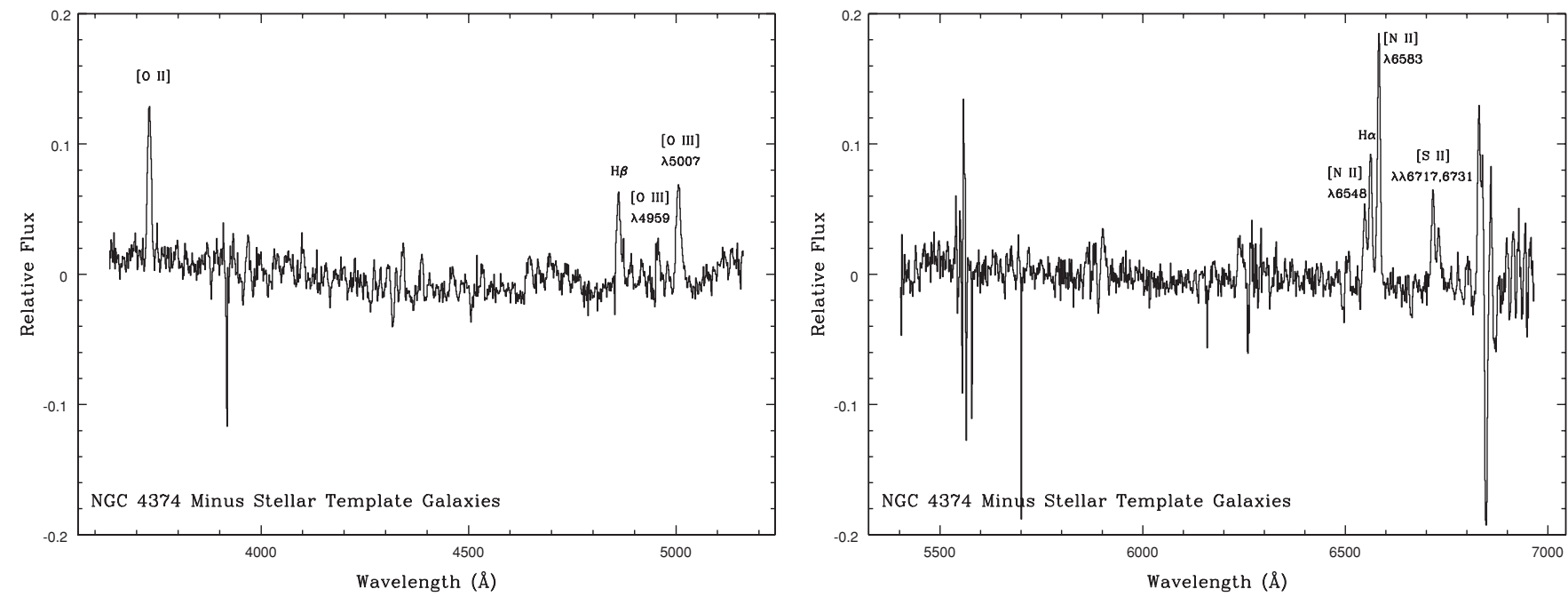

Figure 2. Blue (left) and red (right) spectra of NGC4374 with template galaxies subtracted, removing the stellar continuum. Large residuals at 5500 and $6800 \AA$ are due to different night sky line positions in the rest wavelength of NGC4374 and template galaxies. The large residual at $3900 \AA$ is due to the imperfect matching between the Ca H\&K lines of the template galaxies and NGC 4374.

without any detected warm ISM is an involved and iterative process. First, the $\mathrm{H} \alpha$ and $[\mathrm{N}$ II] $] 26583$ region of the spectra is examined for obvious emission. Using the local continuum, upper limits on the line fluxes are determined for galaxies with no obvious $\mathrm{H} \alpha$ and $[\mathrm{N}$ II] $] 26583$ emission. Ten of the original 21 galaxies match this criteria and act as potential template galaxies. We take these 10 galaxies and combine them into a master template galaxy. When combined into the template galaxy, the strongest of these weak emitters will not be included in the template creation because of the aggressive clipping algorithm in the combination routine. To determine the weak emitters the template is subtracted from the individual galaxy observations and examined for emission lines. Three of the 10 template galaxies revealed oxygen lines and were removed from the master template. This process was repeated with the emitters removed and reveal four more weak emitters, leaving three galaxies without emission line activity: NGC 1407, NGC 3115 , and NGC 3379. As an independent check of our template definition methodology, all seven of the potential template galaxies were subtracted from the galaxy with the strongest emission lines (NGC 2768) and we determined that the three template galaxies selected resulted in the highest reported fluxes (i.e., lowest background).

Previous studies show that the template galaxies (NGC 1407, NGC 3115, and NGC 3379) are frequently observed to be weak emitters or completely void of warm gas. NGC 3379 is reported to have a weak $\mathrm{H} \alpha$ in MFCF but an upper limit below this reported detection is determined in G94-5. NGC 3115 is detected only as an upper limit by MFCF and has been previously used as a stellar template galaxy by the Palomar spectral survey of the nuclear regions of 500 nearby galaxies (Ho et al. 1993). NGC 1407 is marginally detected in both the G94-5 and MFCF studies, but the fluxes reported differ by a factor of 5. The inconsistencies of weak detections in the narrowband surveys reveal intrinsic limitations of the narrowband technique. Broadband red imaging is used to subtract an underlying stellar population from the narrowband data; a process which is susceptible to scaling errors. The stellar template constructed from our sample contains $<10^{-16} \mathrm{erg} \mathrm{s}^{-1} \mathrm{~cm}^{-2} \mathrm{com}-$ bined $\mathrm{H} \alpha$ and $[\mathrm{N}$ II] $] \lambda 6583$ flux; this is an order of magnitude more sensitive as a nondetection than the narrowband imaging surveys.
Once the template galaxies are defined, the stellar population was subtracted from each of the other galaxies, revealing emission lines and any differences in stellar populations. An example subtraction for NGC 4374 is shown in Figure 2. In all galaxies, the residuals in the subtractions are flat with large-scale variation at an $8 \%-10 \%$ level in the blue and better than $5 \%$ in the red. Once the subtractions are made, the relative line fluxes are measured by defining a low-order polynomial to describe the local background and using a Gaussian profile to extract the line flux. A Gaussian decomposition is only necessary in the crowded $[\mathrm{N}$ II $] \lambda 6548, \mathrm{H} \alpha,[\mathrm{N}$ II] $\lambda 6583$ region and the fitting method gives equivalent results to raw counts above a background for all other lines. Note that because of the coarse spectral sampling and the relatively low velocity dispersions, the width of the Gaussian profiles contain no useful kinematic information and is thus not reported. In eight of the nontemplate galaxies, the signal to noise in the data is insufficient for emission line work, or only $\mathrm{H} \alpha$ is observed. For the rest of the sample, extracted relative line fluxes normalized to $\mathrm{H} \beta$ are reported in Table 3. The reported flux ratios have been dereddened based on an average value for the reddening from Burstein \& Heiles (1984) and Schlegel et al. (1998). The observed relative fluxes reported here do not correct for internal galaxy reddening, displaying the Balmer decrements as observed; below we apply the correction (see Section 4.2). The errors reported are determined by calculating the range of acceptable background levels within the noise of the local continuum for isolated lines and combining this background uncertainty in quadrature with the range of acceptable Gaussian widths that reproduce the total observed line flux for the crowded $[\mathrm{N}$ II $]-\mathrm{H} \alpha$ complex.

\section{WARM ISM EMISSION LINE RESULTS}

\subsection{Excitation Mechanism \\ 4.1.1. Normal Galaxies}

There are many possible ionization mechanisms for the warm ISM that have been previously explored and ruled out by the key warm ISM studies (Section 1.2) and other works. Photoionization by hot young stars (Kim 1989; Shields 1991) is inconsistent with both the optical colors (G94-5; MFCF) and integrated optical spectra (Sadler 1987; Heckman et al. 1988). 
Table 3

Observed Nebular Emission Line Ratios Relative to $\mathrm{H} \beta$

\begin{tabular}{|c|c|c|c|c|c|c|c|c|c|c|}
\hline Galaxy & $\begin{array}{l}\text { [O II] } \\
\lambda 3727 \\
\end{array}$ & $\begin{array}{c}\mathrm{Ne} \text { III] } \\
\lambda 3869\end{array}$ & $\begin{array}{c}\mathrm{H} \beta \\
\lambda 4862 \\
\end{array}$ & $\begin{array}{l}\text { [O III] } \\
\lambda 4959\end{array}$ & $\begin{array}{l}\text { [O III] } \\
\lambda 5007 \\
\end{array}$ & $\begin{array}{c}\mathrm{O} \mathrm{I}] \\
\lambda 6300 \\
\end{array}$ & $\begin{array}{l}{\left[\mathrm{N}_{\mathrm{II}}\right]} \\
\lambda 6548 \\
\end{array}$ & $\begin{array}{c}\mathrm{H} \alpha \\
\lambda 6563 \\
\end{array}$ & $\begin{array}{l}\mathrm{N} \text { II }] \\
\lambda 6583 \\
\end{array}$ & $\begin{array}{c}{[\mathrm{S} \text { II }]} \\
\lambda \lambda 6717,6731\end{array}$ \\
\hline NGC 2768 & $70 \pm 7$ & $14 \pm 6$ & $10 \pm 3$ & $1 \pm 4$ & $24 \pm 4$ & $8 \pm 2$ & $20 \pm 4$ & $29 \pm 5$ & $45 \pm 7$ & $50 \pm 8$ \\
\hline NGC 3489 & $19 \pm 5$ & $2 \pm 3$ & $10 \pm 2$ & $2 \pm 5$ & $13 \pm 5$ & $3 \pm 2$ & $30 \pm 8$ & $34 \pm 5$ & $57 \pm 8$ & $37 \pm 7$ \\
\hline NGC 3607 & $32 \pm 8$ & $6 \pm 3$ & $10 \pm 3$ & $7 \pm 3$ & $20 \pm 4$ & $2 \pm 4$ & $25 \pm 5$ & $48 \pm 6$ & $59 \pm 8$ & $22 \pm 5$ \\
\hline NGC 4125 & $74 \pm 8$ & $14 \pm 4$ & $10 \pm 2$ & $23 \pm 5$ & $71 \pm 10$ & $3 \pm 3$ & $19 \pm 4$ & $34 \pm 8$ & $40 \pm 5$ & $23 \pm 7$ \\
\hline NGC 4261 & $16 \pm 4$ & $6 \pm 5$ & $10 \pm 3$ & $6 \pm 2$ & $12 \pm 3$ & $3 \pm 2$ & $15 \pm 5$ & $22 \pm 8$ & $39 \pm 5$ & $15 \pm 6$ \\
\hline NGC 4374 & $16 \pm 4$ & $<1$ & $10 \pm 2$ & $5 \pm 2$ & $12 \pm 4$ & $2 \pm 4$ & $21 \pm 9$ & $35 \pm 6$ & $49 \pm 8$ & $29 \pm 5$ \\
\hline NGC 4636 & $24 \pm 5$ & $2 \pm 4$ & $10 \pm 3$ & $4 \pm 4$ & $13 \pm 3$ & $2 \pm 5$ & $10 \pm 3$ & $33 \pm 5$ & $49 \pm 7$ & $21 \pm 5$ \\
\hline NGC 5044 & $24 \pm 3$ & $2 \pm 3$ & $10 \pm 2$ & $6 \pm 2$ & $20 \pm 3$ & $3 \pm 2$ & $33 \pm 8$ & $32 \pm 6$ & $53 \pm 8$ & $33 \pm 5$ \\
\hline NGC 5846 & $26 \pm 5$ & $2 \pm 3$ & $10 \pm 4$ & $6 \pm 3$ & $14 \pm 4$ & $<1$ & $8 \pm 5$ & $29 \pm 4$ & $36 \pm 8$ & $13 \pm 10$ \\
\hline
\end{tabular}

Ionization from radiation associated with an AGN (Fosbury et al. 1982) is ruled out by the large radial extent of the warm ISM observed in the narrowband imaging (TdSA; G94-5; MFCF). Shock excitation (Ford \& Butcher 1979; Heckman et al. 1989) would produce filamentary ionization regions, where smooth emission is observed (TdSA; G94-5; MFCF). Further, the input energy from shocks is too low by 2 orders of magnitude for typical densities and velocities as noted by Sparks et al. (1989). Condensation out of the hot phase and into warm phase filaments has been investigated for a number of X-ray clusters (Cowie et al. 1980; Hu et al. 1985) but the large number of recombinations required per hydrogen atom (e.g., Johnstone et al. 1987; TdSA and references therein) is inconsistent with the observed recombination rates for early-type galaxies (Sparks et al. 1989).

The three remaining viable excitation mechanisms for the warm ISM are photoionization from PAGB stars, electron conduction from the hot phase (e.g., Sparks et al. 1989), and photoionization by extreme UV (EUV) photons from the hot phase (Voit \& Donahue 1990; Donahue \& Voit 1991). The data in the key warm ISM studies are equally consistent with all of these ionization mechanisms. However, photoionization by PAGB stars is the preferred mechanism since these old stars are known to populate the galaxies and given the significant correlation of $L_{\mathrm{H} \alpha}$ with $L_{\mathrm{B}}$ within a given region (MFCF) strongly indicates a stellar ionizing source. Further, the presence of the far UV flux has been confirmed in a small sample of 30 early-type galaxies with International Ultraviolet Explorer (IUE; Burstein et al. 1988; for a review of far UV flux in earlytype galaxies see O'Connel 1999).

However, to adopt PAGB stars as the ionizing source, excludes the observed correlations between $\mathrm{H} \alpha$ and X-ray luminosity (TdSA; Goudfrooij 1997; MFCF). The correlations between the X-ray and warm ISM morphologies (Singh et al. 1995; Trinchieri et al. 1997; Trinchieri \& Goudfrooij 2002) provide additional support for photoionization of the warm ISM by the EUV flux from the hot ISM, but it is possible to construct physical conditions that reproduce these morphological links (e.g., cooling of the hot ISM). Although there have been detected correlations between the $L_{\mathrm{H} \alpha}$ and $L_{\mathrm{X}} / L_{\mathrm{B}}$, the correlations contain much more scatter and are less significant than the correlation between $L_{\mathrm{H} \alpha}$ with $L_{\mathrm{B}}$ within a given region. The large amount of scatter in the X-ray relationship and the known high $L_{\mathrm{X}} / L_{\mathrm{B}}$ galaxies which contain weak or no $\mathrm{H} \alpha$ emission and strong $\mathrm{H} \alpha$ emitters with low $L_{\mathrm{X}} / L_{\mathrm{B}}$ provide insight that the hot and warm ISM link is more tenuous than the link between the stars and the warm ISM. Finally, the key studies in Section 1.2 and subsequent work (Sarzi et al. 2006) indicate a strong connection between the dust (cold ISM) and the emission line regions, but there are not strong correlations between $L_{\mathrm{FIR}}$ and $L_{\mathrm{X}} / L_{\mathrm{B}}$ in any of the key studies or in recent Spitzer Observations (Temi et al. 2007).

Emission line ratios of the different ionization species have the ability to discriminate between collisionally excitation and photoionization. Comparing our sample's line ratios to line ratios predicted for photoionization models of Allen et al. $(1998,1999)$ and Binette et al. (1996) and shock ionization of Allen et al. (2008), we conclude that the data are consistent with photoionization. Unfortunately, these data are not discriminating due to the large errors in the data and the region populated by these galaxies happens to land in an overlap region for collisional and photoionization.

Additional support for the photoionization of this gas is seen with the Far Ultraviolet Space Explorer (FUSE; Bregman et al. 2005). The observed UV radiation field produces sufficient photons shortward of $912 \AA$ to ionize the warm ISM and is consistent with the number of predicted extreme horizontalbranch stars, or equivalently PAGB stars.

When the data presented here are combined with the literature, it presents a reasonable case for photoionization of the warm ISM. This is the preferred ionization mechanism in the literature and in discriminating between the two sources of photoionization, PAGB stars, or the hot ISM, it would seem more probable that PAGB stars provide the necessary ionizing photons.

\subsubsection{LINER Galaxies}

Four of the galaxies listed in Table 3 are reported as low ionization nuclear emission-line regions (LINERs) galaxies in the literature: NGC 2768 (Heckman 1980), NGC 3115 (Ho et al. 1993), and NGC 4125 and NGC 4261 (Ho et al. 1995). The question of identical treatment of these LINER galaxies and the normal galaxies arises. LINERs are strictly defined to be galaxies with line ratios of $[\mathrm{O}$ II $] \lambda 3727$ greater than $[\mathrm{O}$ III $] \lambda 5007$ and $[\mathrm{O}$ I] $\lambda 6100$ greater than one-third [O III] $\lambda 5007$ (Heckman 1980). While useful for separating classes of objects, the LINER definition as noted by its creator, Heckman, has no physical basis and in reality only provides a crude boundary between photoionization and a low-level AGN activity.

For our purposes, the LINER identification acts as a flag for further investigation, and not as an automatic exclusion filter. The line ratios for the four LINER galaxies are consistent within the photoionization, but the excitation mechanism and thus the valid regions in diagnostic diagrams are still uncertain and being debated for LINERs (for a review of the physical mechanisms behind LINERs see Filippenko 1996 and Barth 2002). 
Although it is not entirely clear what to expect for LINER galaxies, the presence of strong collisionally excited lines casts a cloud of doubt of pure photoionization. The [Ne III] $\lambda 3869$ line is an indicator of the importance of collisional excitation because the collisional cross section is over an order of magnitude larger than the photoionization transition probability for this line. NGC 2768 and NGC 4125 have significant [Ne III] $\lambda 3869$ fluxes and thus we conclude that these systems have a more complex physical structure and excitation mechanism than photoionization. These two galaxies are excluded in the subsequent sections where pure photoionization is assumed. Note that NGC 4125 and NGC 4261 show no distinguishing line ratios from the normal galaxies, and the data presented here do not support the LINER classification. These two galaxies were classified as LINERs by Ho et al. (1995) which had a similar observational aperture as this study, but only had wavelength coverage down to $4200 \AA$, excluding the useful [O II] 33727 information.

\subsection{Oxygen Metallicity}

For those galaxies where photoionization is the dominant ionization mechanism, the line ratios can be used to determine the metallicity of the warm phase gas. There are two critical assumptions to be addressed before using these line ratios and photoionization models to determine global metallicities. The first assumption is that the UV flux from PAGB stars ionizing a diffuse ISM on a galactic scale is well represented by photoionization models that were developed for Strömgren sphere-type $\mathrm{H}$ II regions. First, we note that the models are determining the balance between photoionization by UV photons and recombinations to the ions; this is fundamentally the same equation in a galaxy-wide ISM as an $\mathrm{H}$ II region, given a source of ionizing photons internal to a volume of gas. The UV flux and electron densities of the two environments are similar, although PAGB stars have different spectral shapes than high-mass mainsequence stars, but McGaugh (1991) shows that the photoionization models are largely insensitive to the spectral shape of the ionizing star. The models assume that the gas is ejected from the ionizing star (and thus of the same metallicity), this is a feature used to calibrate the observed line ratios, but a direct link between the ionizing source and the ejecta is not a necessary condition to employ the models. We argue that the photoionization models will be applicable to the warm ISM, but we readily acknowledge that there are differences in these two environments which will lead to greater uncertainties in its application here.

The second key assumption is that the analysis of the linear combination of many individual diffuse ionized regions observed in an integrated galaxy spectra produces a result that is indicative of the mean properties of the observed regions. Kobulnicky et al. (1999) conducted a study addressing these issues in nearby galaxies, observing $\mathrm{H}$ II regions individually through small apertures and globally with drift scanning with a long slit. The authors conclude that global metallicity determinations, accurate to 0.2 dex, are possible with long-slit integrated spectra.

Metallicity determinations of ionized gas in individual H II regions are built upon photoionization models, determining ratios of ionized species relative to hydrogen. The most common diagnostic is the $R 23$ parameterization, $R 23 \equiv$ (([O II $] \lambda 3727+[\mathrm{O}$ III $] \lambda \lambda 4959,5007) / \mathrm{H} \beta)$, defined by Pagel et al. (1979) and refined by McGaugh (1991). This parameterization is convenient since it employs the readily available optical oxygen lines; however, it requires the $[\mathrm{O}$ II $] \lambda 3727 /[\mathrm{N}$ II $] \lambda 6583$
Table 4

R23 Parameter and Oxygen Metallicity

\begin{tabular}{lccc}
\hline \hline Galaxy & $\log \mathrm{R} 23$ & {$[\mathrm{O} / \mathrm{H}]$} & $Z_{\text {Oxygen }} / Z_{\odot}$ \\
\hline NGC 3489 & $0.63_{-0.14}^{+0.11}$ & $-3.26 \pm 0.2$ & $1.13_{-0.30}^{+0.49}$ \\
NGC 3607 & $0.93_{-0.11}^{+0.08}$ & $-3.66 \pm 0.2$ & $0.43_{-0.18}^{+0.29}$ \\
NGC 4261 & $0.51_{-0.07}^{+0.06}$ & $-3.14 \pm 0.2$ & $1.44_{-0.29}^{+0.47}$ \\
NGC 4374 & $0.58_{-0.10}^{+0.09}$ & $-3.20 \pm 0.2$ & $1.26_{-0.31}^{+0.50}$ \\
NGC 4636 & $0.68_{-0.11}^{+0.09}$ & $-3.31 \pm 0.2$ & $0.98_{-0.26}^{+0.41}$ \\
NGC 5044 & $0.75_{-0.06}^{+0.05}$ & $-3.37 \pm 0.2$ & $0.86_{-0.24}^{+0.36}$ \\
NGC 5846 & $0.68_{-0.09}^{+0.08}$ & $-3.30 \pm 0.2$ & $1.00_{-0.26}^{+0.37}$ \\
\hline
\end{tabular}

information to resolve a degeneracy present in the $R 23$ models.

We take the line ratios reported in Table 3 and correct for reddening internal to the galaxy using the interstellar extinction curve of Savage \& Mathis (1979) and assuming case B ratio for the $\mathrm{H} \alpha$ to $\mathrm{H} \beta$ at $T=10^{4}$ (Osterbrock 1989). For NGC 4261 no reddening correction is applied, as the Balmer decrement indicates a nonphysical negative reddening.

These dereddened lines are used to generate $R 23$ values for the galaxies. Because of the faint nature of the $[\mathrm{O}$ III $] \lambda 4959$ line, we used the conical, equilibrium value of $1 / 3$ the line flux of [O III] $\lambda 5007$ when this line was buried in the noise of the subtracted spectra. All of the galaxies end up on the upper branch of the $R 23$ curve as indicated by the [O $\mathrm{II}] \lambda 3727 /[\mathrm{N} \mathrm{II}] \lambda 6583$ diagnostic. To determine metallicities, we employ McGaugh (1991) calibration as parameterized by Kobulnicky et al. (1999) and reported the metallicity results in Table 4 . For the zero point of the solar metallicity we employ a solar photospheric oxygen abundance of $[\mathrm{O} / \mathrm{H}]=-3.3$ (Asplund et al. 2005; Scott et al. 2009), but note the uncertainty and current debate about the solar determinations. The errors reported on the oxygen metallicities in these galaxies are at least 0.2 dex as these are the dominating and intrinsic uncertainties in the models used to create the $R 23$ grid and its application to global galaxy spectra (see Kobulnicky et al. 1999). The median metallicity for the seven early-type galaxies is $\mathrm{O} / \mathrm{O}_{\odot}=1.01$. Within these $0.2 \mathrm{dex}$ absolute errors, none of the galaxies are significantly different from one another.

\section{IMPLICATIONS AND DISCUSSION}

The determined mean oxygen metallicity of solar for the warm phase of the ISM has implications for the origin of this gas and its relationship to other phases of the ISM and the stellar population. The limitations of this study are apparent with a small sample of seven galaxies, large 0.2 dex errors, and a less than ideal application of $\mathrm{H}$ II region models to the warm ISM. However, this work does present new information as it appears that there are no determination of the metallicity of the warm ISM in early-type galaxies in the literature. Sarzi et al. (2006) conducted the most recent large survey of ionized gas in early-type galaxies and conclude at the end of the study that the origin of the ionized gas is still unsolved and state that the metallicity provides an insightful clue. Below we consider the implications of a near solar metallicity for the warm ISM.

\subsection{Origin of the Warm ISM}

A warm ISM with a solar metallicity argues against an external origin model for the emission line gas. The accretion galaxies involved in minor mergers are presumably dwarf irregular (dIrr) galaxies, since dwarf spheroidal (dSph) galaxies 
are most frequently devoid of gas. Gaseous and dusty galaxies with near solar metallicity are luminous (greater than $10^{10} L_{\odot}$ ) and massive (Garnett 2002). Mergers of these types of objects produce profound disturbances, which are simply not observed in these early-type galaxies with $\mathrm{H} \alpha$ emission. It is possible that an intense gas enrichment by supernovae type II occurs once low metallicity gas is accreted; however, the broadband colors and global galaxy spectra (G94-5; MFCF) show little evidence for major star formation. Further, the observed supernova rates are too low to account for such a drastic enrichment (Cappellaro et al. 1999).

We can compare a solar metallicity for the warm ISM with metallicities of the hot ISM and the stars. The stellar population in early-type galaxies is generally observed to have a solar or supersolar metallicity (Trager et al. 2000a, 2000b). A number of the seven galaxies observed in this work have stellar $\mathrm{Fe}$ and $\alpha$ metallicity measurements in the literature, although there are some inconsistencies between authors (NGC 4261 is measured to have an $[\mathrm{Fe} / \mathrm{H}]$ of $-0.03,0.275,0.29$, and 0.188 by Trager et al. 2000b, Thomas et al. 2005, Howell 2005, and SánchezBlázquez et al. 2006, respectively.) Not all the galaxies have measured stellar metallicities but a five galaxy average from all of the determinations in the 2005 and 2006 papers listed above is $[\mathrm{Fe} / \mathrm{H}]=0.22$, ranging from $[\mathrm{Fe} / \mathrm{H}]=0.099$ to $[\mathrm{Fe} / \mathrm{H}]=0.44$ $([\alpha / \mathrm{H}]$ is similar). It is important to remember that Trager et al. (2000a) estimate the absolute error on stellar metallicity determinations close to a factor of 2 , while the relative errors are much smaller. The Chandra X-ray Observatory has the spatial resolution to resolve and remove the stellar component from the gas which is leading to better data and a revision of our (mis)understanding of the metallicity in the hot ISM. Recently, Humphrey \& Buote (2006) analyzed a sample of 28 early-type galaxies observed with Chandra and determined that the hot ISM abundances are consistent and perhaps higher than the stellar abundances. In summary, a $0.50-1.50$ solar metallicity in the warm ISM is consistent with both hot ISM and stellar metallicity determinations.

It has been thought that early-type galaxies would have a problem generating and retaining dust and warm gas, due to the hostile conditions provided by the hot ISM and the low injection rate of new material into the ISM from stars. Parriott \& Bregman (2008) have shown that it is possible for dust to condense in the circumstellar envelopes of AGB stars and then subsequently be injected into the hot ISM. Although this dust has a short lifetime in the hot ISM, the injection rate has been observed to be greater than the destruction rate and could account for the total observed mass, given a simple evolution of this injection rate as more massive stars evolved off the main sequence in the past (Athey et al. 2002). An internal origin for the dust is supported by the G94-5 and MFCF studies indicating that the majority $(\sim 90 \%)$ of the dust is distributed smoothly throughout the galaxy. The internal origin for the cold ISM is further confirmed by a $2.29 \mu \mathrm{m} \mathrm{CO}$ absorption feature study that finds a lower velocity dispersion for the dust than that of the stars in a sample of 25 nearby early-type galaxies (Silge \& Gebhardt 2003). Finally from the analysis of Spitzer observations of 46 galaxies in the FIR $(24,70$, and $160 \mu \mathrm{m})$, Temi et al. (2007) propose an AGB mass loss, internal origin for the observed dust, which sinks to the core of the galaxy and is periodically driven out by AGN activity. This outward transport of dust may cool the hot gas down to the warm phase.

The major stumbling block to a galaxy internal origin for dust and gas is the observations of gas kinematics which are distinct with respect to the stars. Recent observational evidence has suggested that there may be different evolutionary tracks for early-type galaxies. The classification of early-type galaxies into fast and slow rotation categories by Emsellem et al. (2007) and the key ingredient of gas for the formation and evolution of fast rotators provides an important dividing line for future discussions on the origin of the ISM in early-type galaxies.

The authors acknowledge useful discussions with P. Goudfrooij and E. D. Miller. We also thank an anonymous referee for suggestions that significantly improved this paper.

\section{REFERENCES}

Aaronson, M. 1986, in Star Forming Dwarf Galaxies and Related Objects, ed. D. Kunth, J. Thanh Van Tran, \& T. Xuan Thuan (Paris: Atlantica Séguier Frontièrs), 125

Allen, M. G., Dopita, M. A., \& Tsvetanov, Z. I. 1998, ApJ, 493, 571

Allen, M. G., Dopita, M. A., Tsvetanov, Z. I., \& Sutherland, R. S. 1999, ApJ, 511,686

Allen, M. G., Groves, B. A., Dopita, M. A., Sutherland, R. S., \& Kewley, L. J. 2008, ApJS, 178, 20

Asplund, M., Grevesse, N., \& Sauval, A. J. 2005, in ASP Conf. Ser. 336, Cosmic Abundances as Records of Stellar Evolution and Nucleosynthesis, ed. T. G. Barnes. III \& F. N. Bash (San Francisco, CA: ASP), 25

Athey, A., Bregman, J. N., Bregman, J. D., Temi, P., \& Sauvage, M. 2002, ApJ, 571,272

Barth, A. J. 2002, in ASP Conf. Ser. 258, Issues in Unification of Active Galactic Nuclei, ed. R. Maiolino, A. Marconi, \& N. Nagar (San Francisco, CA: ASP), 147

Binette, L., Wilson, A. S., \& Storchi-Bergmann, T. 1996, A\&A, 312, 365

Bregman, J. N. 1978, ApJ, 224, 768

Bregman, J. N., Miller, E. D., Athey, A. E., \& Irwin, J. A. 2005, ApJ, 635, 1031

Brown, B. A., \& Bregman, J. N. 1998, ApJ, 495, L75

Burstein, D., Bertola, F., Buson, L. M., Faber, S. M., \& Lauer, T. R. 1988, ApJ, 328,440

Burstein, D., \& Heiles, C. 1984, ApJS, 54, 33

Caldwell, N. 1984, PASP, 96, 287

Caon, N., Macchetto, D., \& Pastoriza, M. 2000, ApJS, 127, 39

Cappellaro, E., Evans, R., \& Turatto, M. 1999, A\&A, 351, 459

Cowie, L. L., Fabian, A. C., \& Nulsen, P. E. J. 1980, MNRAS, 191, 399

Demoulin-Ulrich, M.-H., Butcher, H. R., \& Boksenberg, A. 1984, ApJ, 285 527

de Vaucouleurs, G., de Vaucouleurs, A., Corwin, H. G., Buta, R. J., Paturel, G., \& Fouque, P. 1991, Third Reference Catalogue of Bright Galaxies (Berlin: Springer-Verlag)

Donahue, M., \& Voit, G. M. 1991, ApJ, 381, 361

Draine, B. T., \& Salpeter, E. E. 1979, ApJ, 231, 438

Emsellem, E., et al. 2007, MNRAS, 379, 401

Faber, S. M., \& Gallagher, J. S. 1976, ApJ, 204, 365

Faber, S. M., Wegner, G., Burstein, D., Davies, R. L., Dressler, A., Lynden Bell, D., \& Terlevich, R. J. 1989, ApJS, 69, 763

Fabian, A. C. 1991, in IAU Symp. 144, The Interstellar Disk-Halo Connection in Galaxies, ed. H. Bloemen (Dordrecht: Kluwer), 237

Ferrari, F., Pastoriza, M. G., Macchetto, F. D., Bonatto, C., Panagia, N., \& Sparks, W. B. 2002, A\&A, 389, 355

Ferrari, F., Pastoriza, M. G., Macchetto, F., \& Caon, N. 1999, A\&AS, 136, 269

Filippenko, A. V. 1996, in ASP Conf. Ser. 103: The Physics of Liners in View of Recent Observations, ed. M. Eracleous et al. (San Francisco, CA: ASP), 17

Ford, H. C., \& Butcher, H. 1979, ApJS, 41, 147

Forman, W., Schwarz, J., Jones, C., Liller, W., \& Fabian, A. C. 1979, ApJ, 234, L27

Fosbury, R. A. E., et al. 1982, MNRAS, 201, 991

Garnett, D. R. 2002, ApJ, 581, 1019

Goudfrooij, P. 1994, PhD thesis, Univ. Amsterdam

Goudfrooij, P. 1995, PASP, 107, 502

Goudfrooij, P. 1997, in ASP Conf. Ser. 116, The Nature of Elliptical Galaxies, 2nd Stromlo Symposium, ed. M. Arnaboldi, G. S. Da Costa, \& P. Saha (San Francisco, CA: ASP), 338

Goudfrooij, P., \& de Jong, T. 1995, A\&A, 298, 784

Goudfrooij, P., de Jong, T., Hansen, L., \& Norgaard-Nielsen, H. U. 1994a, MNRAS, 271, 833 
Goudfrooij, P., Hansen, L., Jorgensen, H. E., \& Norgaard-Nielsen, H. U. 1994b, A\&AS, 105, 341

Goudfrooij, P., Hansen, L., Jorgensen, H. E., Norgaard-Nielsen, H. U., de Jong, T., \& van den Hoek, L. B. 1994c, A\&AS, 104, 179

Heckman, T. M. 1980, A\&A, 87, 152

Heckman, T. M., Baum, S. A., van Breugel, W., \& McCarthy, P. J. 1988, in NATO ASIC Proc. 229, Cooling Flows in Clusters and Galaxies, ed. A. Fabian (Cambridge: Cambridge Univ. Press), 245

Heckman, T. M., Baum, S. A., van Breugel, W. J. M., \& McCarthy, P. 1989, ApJ, 338,48

Ho, L. C., Filippenko, A. V., \& Sargent, W. L. W. 1993, ApJ, 417, 63

Ho, L. C., Filippenko, A. V., \& Sargent, W. L. 1995, ApJS, 98, 477

Howell, J. H. 2005, AJ, 130, 2065

Hu, E. M., Cowie, L. L., \& Wang, Z. 1985, ApJS, 59, 447

Humphrey, P. J., \& Buote, D. A. 2006, ApJ, 639, 136

Johnstone, R. M., Fabian, A. C., \& Nulsen, P. E. J. 1987, MNRAS, 224, 75

Kim, D. 1989, ApJ, 346, 653

Knapp, G. R., Gunn, J. E., \& Wynn-Williams, C. G. 1992, ApJ, 399, 76

Kobulnicky, H. A., Kennicutt, R. C., \& Pizagno, J. L. 1999, ApJ, 514, 544

Lequeux, J., Rayo, J. F., Serrano, A., Peimbert, M., \& Torres-Peimbert, S. 1979, A\&A, 80, 155

Macchetto, F., Pastoriza, M., Caon, N., Sparks, W. B., Giavalisco, M., Bender, R., \& Capaccioli, M. 1996, A\&AS, 120, 463

Massey, P., Strobel, K., Barnes, J. V., \& Anderson, E. 1988, ApJ, 328, 315

Mathews, W. G., \& Baker, J. C. 1971, ApJ, 170, 241

Mayall, N. U. 1936, PASP, 48, 14

Mayall, N. U. 1958, in IAU Symp. 5, Comparison of the Large-Scale Structure of the Galactic System with that of Other Stellar Systems, ed. N. Grace (Dordrecht: Kluwer), 23

McGaugh, S. S. 1991, ApJ, 380, 140

O'Connell, R. W. 1999, ARA\&A, 37, 603

Osterbrock, D. E. 1989, Astrophysics of Gaseous Nebulae and Active Galactic Nuclei (Mill Valley, CA: University Science Books)
O'Sullivan, E., Forbes, D. A., \& Ponman, T. J. 2001, MNRAS, 328, 461

Pagel, B. E. J., Edmunds, M. G., Blackwell, D. E., Chun, M. S., \& Smith, G. 1979, MNRAS, 189, 95

Parriott, J. R., \& Bregman, J. N. 2008, ApJ, 681, 1215

Phillips, M. M., Jenkins, C. R., Dopita, M. A., Sadler, E. M., \& Binette, L. 1986, AJ, 91, 1062

Sadler, E. M. 1987, in IAU Symp. 127: Structure and Dynamics of Elliptical Galaxies, ed. P. T. de Zeeuw (Dordrecht: Kluwer), 125

Sánchez-Blázquez, P., Gorgas, J., Cardiel, N., \& González, J. J. 2006, A\&A, 457,809

Sandage, A. 1957, ApJ, 125, 422

Sarzi, M., et al. 2006, MNRAS, 366, 1151

Savage, B. D., \& Mathis, J. S. 1979, ARA\&A, 17, 73

Schlegel, D. J., Finkbeiner, D. P., \& Davis, M. 1998, ApJ, 500, 525

Scott, P., Asplund, M., Grevesse, N., \& Sauval, A. J. 2009, ApJ, 691, L119

Shields, J. C. 1991, AJ, 102, 1314

Silge, J. D., \& Gebhardt, K. 2003, AJ, 125, 2809

Singh, K. P., Bhat, P. N., Prabhu, T. P., \& Kembhavi, A. K. 1995, A\&A, 302, 658

Sparks, W. B., Macchetto, F., \& Golombek, D. 1989, ApJ, 345, 153

Temi, P., Brighenti, F., \& Mathews, W. G. 2007, ApJ, 660, 1215

Thomas, D., Maraston, C., Bender, R., \& Mendes de Oliveira, C. 2005, ApJ, 621,673

Trager, S. C., Faber, S. M., Worthey, G., \& González, J. J. 2000a, AJ, 120, 165

Trager, S. C., Faber, S. M., Worthey, G., \& González, J. J. 2000b, AJ, 119, 1645

Trinchieri, G., \& di Serego Alighieri, S. 1991, AJ, 101, 1647

Trinchieri, G., \& Goudfrooij, P. 2002, A\&A, 386, 472

Trinchieri, G., Noris, L., \& di Serego Alighieri, S. 1997, A\&A, 326, 565

Veron-Cetty, M.-P., \& Veron, P. 1986, A\&AS, 66, 335

Voit, G. M., \& Donahue, M. 1990, ApJ, 360, L15

White, R. E., \& Chevalier, R. A. 1983, ApJ, 275, 69 